\title{
AN EXOTIC INVARIANT FOR 6-MANIFOLDS: THE DIRECT CONSTRUCTION
}

\author{
A. V. ZHUBR
}

\begin{abstract}
Some of the author's previous works, dealing with the classification problem for simply connected closed 6-manifolds, contain a construction of a certain "exotic" invariant $\Gamma$. This construction is substantially indirect and based on nontrivial calculations. In the present paper, a direct construction is suggested, which does not depend on the calculations mentioned and involves only some simple surgery, plus some well-known identities for Stiefel-Whitney and Pontryagin classes, namely, "modulo 2" and "modulo 4" Wu formulas.
\end{abstract}

\section{INTRODUCTION}

In 1, 2], for each simply connected closed 6-manifold $M$, the author introduced an invariant $\Gamma(M)$ of a rather specific kind: this is a family of functions

$$
\left\{\Gamma_{a}(M): H^{2}(M ; \mathbb{Z} / 2 a) \rightarrow \mathbb{Z} / a\right\}, \quad a=0,1,2, \ldots,
$$

defined for $\omega \in H^{2}(M ; \mathbb{Z} / 2 a)$ that satisfy the "spin condition" $\omega \equiv w_{2}(M) \bmod 2$ and also some "consistency axioms" (for the details, see the papers cited above and $\S 1$ below). Along with all the "conventional" invariants (homology, operations, characteristic classes), the new invariant, according to [2, makes up a complete set for the above class of manifolds: any isomorphism between the sets of invariants for two such manifolds is induced by a homeomorphism (or diffeomorphism if the manifolds happen to be smooth). By 22] again, $\Gamma(M)$ is not determined by the tangential homotopy type of $M$ (i. e., its homotopy type plus the tangent bundle); therefore it is not reducible to "conventional" invariants.

The construction of $\Gamma$ given in [2] is, in a sense, "not quite satisfactory": the existence of the family $\left\{\Gamma_{a}\right\}$ is deduced there from information about some 6 -dimensional bordism groups; in its turn, this information is obtained as a result of fairly nontrivial calculations. Thus, it would be desirable to have a more direct construction, not depending on calculations, and dealing with the individual manifold $M$. Such a construction is the subject of this paper. Its idea originated from one long-ago conversation with Volodya Turaev (whom the author has the pleasure to thank); however, some technical difficulties persisted until recently.

The paper consists of 3 sections. In $\S 1$ we provide a brief exposition of our original approach to $\Gamma$, close to that in [1, 2]: we set a list of basic properties (or axioms), derive some more or less immediate consequences of these axioms, and outline the idea of the existence proof in 2. Section 2 is central - here we present our direct construction.

2000 Mathematics Subject Classification. Primary 57N15, 57R55.

Key words and phrases. 6-manifold, classification, surgery, direct construction, invariant.

This work is partially supported by the program "Problems in non-linear dynamics" of the Presidium of Russian Academy of Sciences. 
Finally, in $\S 3$ we consider the problem of proving the properties of $\Gamma$ on the basis of our direct definition.

Our approach works in both DIFF and TOP categories (i.e., for both smooth and topological manifolds); recall that, from a classification point of view, the PL category in dimension 6 can be viewed as indistinguishable from DIFF. For technical reasons, we treat the DIFF case as "primary", giving necessary remarks concerning ToP whenever needed.

\section{$\S 1$. The Invariant $\Gamma$ : Formal Description And some CONSEQUEnCES}

Notation and preliminary remarks. In what follows, $\widehat{\mathbb{N}}$ denotes the set $\{0\} \cup \mathbb{N}=$ $\{0,1,2, \ldots\}$; for $a \in \widehat{\mathbb{N}}$, the cyclic group $\mathbb{Z} / a \mathbb{Z}$ is denoted by $\mathbb{Z}_{a}$ (in particular, $\mathbb{Z}_{0}=\mathbb{Z}$ is the group of integers). The elements of $\mathbb{Z}_{a}$ with $a>0$ can be written as $\overline{0}, \overline{1}$, etc.

For $a \in \widehat{\mathbb{N}}$ and $b \in \mathbb{N}$, the standard injection $\mathbb{Z}_{a} \rightarrow \mathbb{Z}_{a b}$ (multiplication by $b$ ) is denoted by $i_{b}$, and the standard projection $\mathbb{Z}_{a b} \rightarrow \mathbb{Z}_{b}$ (reduction modulo $b$ ) by $\rho_{b}$. The same notation is used for various induced homomorphisms: we write, e.g., $i_{b}: G \otimes \mathbb{Z}_{a} \rightarrow$ $G \otimes \mathbb{Z}_{a b}$ or $\rho_{b}: H_{n}\left(X ; \mathbb{Z}_{a b}\right) \rightarrow H_{n}\left(X ; \mathbb{Z}_{b}\right)$, etc.

Let $M$ be a closed oriented $n$-manifold. Recall that any homology class $c \in H_{n-2}(M)$ can be realized by an oriented $(n-2)$-submanifold $A \subset M$ obtained as a transversal preimage of the hyperplane $\mathbb{C} P(N-1) \subset \mathbb{C} P(N)$, with $N$ sufficiently large, under a map $f: M \rightarrow \mathbb{C} P(N) \approx K(\mathbb{Z}, 2)$ representing the Poincaré dual $D^{-1} c \in H^{2}(M)$. We shall also need the relative case of this, which we state below, for convenience, in the form of the following theorem (equally well known, of course).

Theorem 1. Let $M$ be a compact oriented $n$-manifold, $c \in H_{n-2}(M, \partial M)$ a relative homology class, and $B \subset \partial M$ a closed oriented $(n-3)$-submanifold representing the class $\partial c \in H_{n-3}(\partial M)$. Then there exists a compact oriented proper $(n-2)$-submanifold $A \subset M$ with $[A]=c$ and $\partial A=B$.

Remark 1. Since Top transversality for maps is always true [3], all of the above extends immediately to topological manifolds. In what follows, by a Top submanifold we understand a submanifold having a normal Top-bundle (which, in the case of codimension 2 , is the same as having a normal $O$-bundle, in view of the homotopy equivalence $\left.\mathrm{TOP}_{2} \sim \mathrm{O}_{2}\right)$.

Suppose we are given a compact oriented $n$-manifold $M$ and a number $a \in \widehat{\mathbb{N}}$. We say that a cohomology class $\omega \in H^{2}\left(M ; \mathbb{Z}_{2 a}\right)$ is spin if $\rho_{2}(\omega)=w_{2}(M)$. This terminology is justified by the fact that, for $a=0$, the above equation forces any submanifold $A \subset M$ representing the class $D \omega \in H_{n-2}(M, \partial M)$ to be spin, i. e., to satisfy $w_{2}(A)=0$. (Proof: the restriction $\omega \mid A$ is the same as the Euler class $\chi(\nu A)$ of the normal bundle of $A$ in $M$, so that, reducing by 2 , we have $w_{2}(M) \mid A=w_{2}(\nu A)$, whence $w_{2}(A)=w_{2}(\tau A)=0$.) The set of all spin classes in $H^{2}\left(M ; \mathbb{Z}_{2 a}\right)$ is denoted by $H_{\text {spin }}^{2}\left(M ; \mathbb{Z}_{2 a}\right)$, and its Poincaré dual by $H_{n-2}^{\text {spin }}(M, \partial M)$. Note that $H^{2}\left(M ; \mathbb{Z}_{2 a}\right)$ may be empty for some $a$; otherwise $H_{\text {spin }}^{2}\left(M ; \mathbb{Z}_{2 a}\right)$ is a coset of the subgroup $i_{2} H^{2}\left(M ; \mathbb{Z}_{a}\right) \subset H^{2}\left(M ; \mathbb{Z}_{2 a}\right)$.

Now let $M$ be a closed oriented 6-manifold, and $\omega \in H^{2}(M)$ a cohomology class. From Theorem 1 it easily follows that the cobordism class of a submanifold $A \subset M$ representing the dual $D \omega$ is uniquely determined by $\omega$, so that we obtain a map $H^{2}(M) \rightarrow$ $\Omega_{4}^{S O}$ or, composing this with the signature isomorphism $\sigma: \Omega_{4}^{S O} \rightarrow \mathbb{Z}$, simply a map $H^{2}(M) \rightarrow \mathbb{Z}$. This map (not a homomorphism) is the simplest case of virtual signatures of Hirzebruch [4, $\S 9$ ]. For some formal reasons, here instead of the signature $\sigma(A)$, it seems more convenient to take the Pontryagin number $\left\langle p_{1}(A),[A]\right\rangle=3 \sigma(A)$. By analogy, we call this the virtual Pontryagin number and denote it by $p(\omega)$. There is a formula for 
$p(\omega)$ in terms of the classes $\omega$ and $p_{1}(M)$ :

$$
p(\omega)=\left\langle p_{1}(M) \omega-\omega^{3},[M]\right\rangle
$$

(this is immediate from the additivity of $p_{1}$ with respect to the Whitney sum, and from the fact that $\omega \mid A=\chi(\nu A)$; cf. [4).

Remark 2. To extend the virtual Pontryagin number and formula (1) to ToP, two things are needed: (a) the first Top Pontryagin class can be defined as an integral class, not merely a rational one, and (b) this integral topological $p_{1}$ class is additive with respect to the Whitney sum, like its $S O$-analog. Both (a) and (b) follow straightforwardly from the fact that the natural homomorphisms $H^{i}(B S \mathrm{TOP}) \rightarrow H^{i}(B S O)$ are bijective for $i \leq 4$. In its turn, this fact is deduced easily (see [5]) from the following information on the homotopy of BTOP: $\pi_{i} B$ TOP $=\pi_{i} B O$ for $i \neq 4, \pi_{4} B$ TOP $=\pi_{4} B O \oplus \mathbb{Z}_{2}$; see $[6$.

The invariant $l \Gamma$ : Formal description. From now on, to avoid tedious repetitions, we agree about the following: the letter $M$ (possibly with subscripts, etc.) denotes some simply connected closed oriented 6-manifold (unless otherwise specified).

In accordance with [1] (for detailed proofs, see 2]), for each simply connected closed oriented 6-manifold $M$ there exists a family of functions

$$
\Gamma_{a}(M): H_{\text {spin }}^{2}\left(M ; \mathbb{Z}_{2 a}\right) \rightarrow \mathbb{Z}_{a}, a \in \widehat{\mathbb{N}}
$$

(in what follows, the value of $\Gamma_{a}(M)$ at $\omega \in H_{\text {spin }}^{2}\left(M ; \mathbb{Z}_{2 a}\right)$ will be denoted either by $\Gamma(M, \omega)$, or simply by $\Gamma(\omega)$ if $M$ is clear from the context), uniquely determined by the following properties (axioms):

$\Gamma 1$ (homeomorphism invariance) For any orientation-preserving homeomorphism $f$ : $M_{1} \rightarrow M$ and any $\omega \in H_{\text {spin }}^{2}\left(M ; \mathbb{Z}_{2 a}\right)$, we have $\Gamma(M, \omega)=\Gamma\left(M_{1}, f^{*} \omega\right)$.

$\Gamma 2$ (cobordism invariance) Let $W$ be an oriented cobordism between $M$ and $M_{1}$ and let $i: M \rightarrow W, i_{1}: M_{1} \rightarrow W$ be the inclusions. If $\omega \in H_{\mathrm{spin}}^{2}\left(W ; \mathbb{Z}_{2 a}\right)$, then $\Gamma\left(M, i^{*} \omega\right)=\Gamma\left(M_{1}, i_{1}^{*} \omega\right)$.

$\Gamma 3$ (coefficient invariance) If $a \in \widehat{\mathbb{N}}, b \in \mathbb{N}$, and $\omega \in H_{\mathrm{spin}}^{2}\left(M ; \mathbb{Z}_{2 a b}\right)$, then $\Gamma\left(\rho_{2 b} \omega\right)=$ $\rho_{b} \Gamma(\omega)$.

$\Gamma 4$ (normalization) If $\omega \in H_{\mathrm{spin}}^{2}(M)$, then $8 \Gamma(\omega)=p(\omega)$.

Note that, by formula (11) and the paragraph before it, axiom $\Gamma 4$ can be rewritten in the form

$$
\Gamma(\omega)=\frac{\left\langle p_{1}(M) \omega-\omega^{3},[M]\right\rangle}{8}=\frac{3 \sigma(A)}{8},
$$

where $A \subset M$ is any submanifold representing the class $D(\omega) \in H_{4}(M)$ (recall that divisibility by 8 follows here from the condition that the class $\omega$ is spin).

A fundamental formula. The above properties allow us to calculate $\Gamma(M, \omega)$ by using some simple surgery (eliminating torsion in $H_{*}(M)$ ) and formula (2). Now we consider this in more detail.

First, suppose that Tors $H_{2}(M)=0$. In this case no surgery is needed: any class $\omega \in H_{\text {spin }}^{2}\left(W ; \mathbb{Z}_{2 a}\right)$ can be represented in the form $\rho_{2 a} \widetilde{\omega}$ for some $\widetilde{\omega} \in H_{\text {spin }}^{2}(M)$, and axioms $\Gamma 3$ and $\Gamma 4$ imply that

$$
\Gamma(M, \omega)=\rho_{a} \Gamma(M, \widetilde{\omega})=\rho_{a}\left(\frac{\left\langle p_{1}(M) \widetilde{\omega}-\widetilde{\omega}^{3},[M]\right\rangle}{8}\right) .
$$

Thus, we see that for Tors $H_{2}(M)=0$ there is no "exotic" invariant: $\Gamma(M)$ is merely a combination of "conventional" invariants. 
Let Tors $H_{2}(M)$ be nonzero. We represent some basis for Tors $H_{3}(M) \approx$ Tors $H_{2}(M)$ by disjoint embedded 3 -spheres with (arbitrarily chosen) normal framings and perform the corresponding spherical modifications. For the resulting manifold $\widetilde{M}$ we have Tors $H_{2}(\widetilde{M})=0$; this $\widetilde{M}$ will be called a resolution manifold for $M$ (evidently, this construction is highly nonunique). Let $W$ be the natural cobordism between $M$ and $\widetilde{M}$. Homotopically, $W$ can be viewed as a result of attaching some 4-cells to $M$ or, dually, some 3 -cells to $\widetilde{M}$. Therefore, we have a canonical isomorphism

$$
i_{*}: H_{2}(M) \rightarrow H_{2}(W)
$$

and a canonical epimorphism

$$
j_{*}: H_{2}(\widetilde{M}) \rightarrow H_{2}(W)
$$

(both induced by the corresponding inclusions). Having this in mind, we can write

$$
H^{2}(M ; G)=H^{2}(W ; G) \subset H^{2}(\widetilde{M} ; G)
$$

for any coefficients $G$; in particular, we can view the class $\omega$ as belonging to each of these three groups (with $G=\mathbb{Z}_{2 a}$ ). By $\Gamma 2$, then we have $\Gamma(M, \omega)=\Gamma(\widetilde{M}, \omega)$. Combining this with formula (3) applied to the manifold $\widetilde{M}$, we obtain our fundamental formula

$$
\Gamma(M, \omega)=\rho_{a} \Gamma(\widetilde{M}, \widetilde{\omega})=\rho_{a}\left(\frac{\left\langle p_{1}(\widetilde{M}) \widetilde{\omega}-\widetilde{\omega}^{3},[\widetilde{M}]\right\rangle}{8}\right)
$$

(where $\widetilde{\omega} \in H^{2}(\widetilde{M})$ is an arbitrary integral class satisfying $\rho_{2 a} \widetilde{\omega}=\omega$ ). Note that the only difference between this formula and (3) is that, instead of $M$, now the right-hand side contains its "resolution" $\widetilde{M}$.

Remark 3. To transfer the above argument to Top, we only need to know that the general position and the "Whitney trick" for elimination of double points work in this category; see [7, Essay III].

The "odd component" of the invariant $\Gamma$. From (5) it easily follows that all $a$ having odd factors can be skipped; in other words, it suffices to restrict ourselves to $a=0$ and $a=2^{k}, k=1,2, \ldots$, only. Indeed, if we take $a=2^{k} b$ with $b$ odd and reduce (5) modulo $b$, then the resulting expression for $\rho_{b} \Gamma(M, \omega)$ can be written as $\overline{8}^{-1}\left(\left\langle p_{1}(\widetilde{M}) \rho_{b} \omega-\rho_{b} \omega^{3},[\widetilde{M}]\right\rangle\right)$ (we use the invertibility of $\overline{8}$ in $\mathbb{Z}_{b}$ ). Now, the expression $\left\langle p_{1}(\widetilde{M}) \rho_{b} \omega-\rho_{b} \omega^{3},[\widetilde{M}]\right\rangle$ is (evidently) cobordism-invariant, so we can replace the "resolution" $\widetilde{M}$ with the original $M$. Thus, $\rho_{b} \Gamma(M, \omega)$ turns out to be a combination of "conventional" invariants, and thereby, negligible in our context.

The invariant $\Gamma$ and the Kirby-Siebenmann class. Yet another consequence of (5) is a relation between $\Gamma(M, \omega)$ and the Kirby-Siebenmann class

$$
\Delta(M)=\Delta\left(\tau_{M}\right) \in H^{4}\left(M ; \mathbb{Z}_{2}\right)
$$

(an obstruction to covering the Gaussian map $\tau: M \rightarrow B$ Top by a map to BO).

Theorem 2. For any $\omega \in H_{\text {spin }}^{2}\left(M ; \mathbb{Z}_{4}\right)$, we have

$$
\Gamma(M, \omega)=\left\langle\Delta(M) w_{2}(M),[M]\right\rangle .
$$

(Note that here the expression $\left\langle\Delta w_{2},[M]\right\rangle$ is the only possibly nontrivial characteristic number for oriented 6-manifolds, because the map $\Delta w_{2}: \Omega_{6}^{S T O P} \rightarrow \mathbb{Z}_{2}$ is an isomorphism; see [6, Proposition 11.2].) 
Proof. For $a=2$, the right-hand side of (5) can be written as $\rho_{2}\left(\frac{1}{8} \sigma(A)\right)$, where $A \subset \widetilde{M}$ represents the class $D \widetilde{\omega}$. As is well known, for any closed spin 4-manifold $A$ its KirbySiebenmann class and its intersection form are related by $\langle\Delta(A),[A]\rangle=\rho_{2}\left(\frac{1}{8} \sigma(A)\right)$ (the proof is given in [6, p. 152]; on the other hand, this follows directly from the existence of such an $A$ with $\sigma(A)=8$; see [8]). Now we observe that $\Delta(A)$ is the same as the restriction of $\Delta(\widetilde{M})$ to $A$, due to the linearity of the normal bundle of $A$ in $\widetilde{M}$ (see Remark 1 above); therefore,

$$
\langle\Delta(A),[A]\rangle=\langle\Delta(\widetilde{M}), D \widetilde{\omega}\rangle=\langle\Delta(\widetilde{M}) \widetilde{\omega},[\widetilde{M}]\rangle=\left\langle\Delta w_{2}(\widetilde{M}),[\widetilde{M}]\right\rangle .
$$

Finally, $\left\langle\Delta w_{2}(\widetilde{M}),[\widetilde{M}]\right\rangle=\left\langle\Delta w_{2}(M),[M]\right\rangle$, because $M$ and $\widetilde{M}$ are in the same cobordism class.

Example: Two manifolds distinguished by $\boldsymbol{\Gamma}$. Let $f: S^{3} \rightarrow S^{6}$ be a smooth embedding representing the generator of the Haefliger knot group $C_{3}^{3} \approx \mathbb{Z}[9$. We choose the "trivial" normal framing (which turns into the standard framing of a flat 3 -sphere in $S^{6}$ under an unknotting regular homotopy), and perform surgery on $S^{6}$ along $f\left(S^{3}\right)$; let $\widetilde{M}$ be the resulting manifold. It is easily seen that $\widetilde{M}$ has the same (additive) homology as $S^{2} \times S^{4}$, and the same (i. e., trivial) stable tangent bundle (by our choice of a normal framing), whence $w_{2}(\widetilde{M})=p_{1}(\widetilde{M})=0$. If $\widetilde{\alpha}$ is a generator of $H^{2}(\widetilde{M}) \approx \mathbb{Z}$, then (with an appropriate choice of $\widetilde{\alpha}$ ) we have $\left\langle\widetilde{\alpha}^{3},[\widetilde{M}]\right\rangle=6$ (see, e.g., [10, Theorem 4]; alternatively, this can be calculated directly, by using Haefliger's explicit construction for $f$ ). With $\widetilde{\omega}=2 \widetilde{\alpha} \in H_{\text {spin }}^{2}(\widetilde{M})$, formula (2) gives $\Gamma(\widetilde{\omega})=-6$.

Now we modify $\widetilde{M}$ and turn $H_{2}(\widetilde{M})$ into a finite group. We represent 4 times the generator of $H_{2}(\widetilde{M})$ by an embedding $g: S^{2} \rightarrow \widetilde{M}$ and perform the corresponding surgery. Let $M$ denote the resulting manifold; from the construction it follows that $H_{2}(M) \approx H_{3}(M) \approx \mathbb{Z}_{4}$. Furthermore, $M$ inherits stable parallelizability (by $\pi_{2} S O=0$ ), so that $w_{2}(M)=p_{1}(M)=0$.

Note that, here, $M$ and $\widetilde{M}$ are in precisely the same relation to each other as in our argument preceding formula (5) (the "inverse" surgery, killing the group $H_{3}(M)$, is to be done along a small 3-sphere linked once with $\left.g\left(S^{2}\right)\right)$. The identifications (4), taken with $G=\mathbb{Z}_{4}$ and $G=\mathbb{Z}_{8}$, yield

$$
\begin{aligned}
& H^{2}\left(M ; \mathbb{Z}_{4}\right)=H^{2}\left(\widetilde{M} ; \mathbb{Z}_{4}\right) \approx \mathbb{Z}_{4}, \\
& H^{2}\left(M ; \mathbb{Z}_{8}\right) \approx \mathbb{Z}_{4} \subset H^{2}\left(\widetilde{M} ; \mathbb{Z}_{8}\right) \approx \mathbb{Z}_{8} .
\end{aligned}
$$

Having this in mind, we take $\alpha=\rho_{4} \widetilde{\alpha}$ and $\omega=i_{2} \alpha$ as generators of the groups $H^{2}\left(M ; \mathbb{Z}_{4}\right)$ and $H^{2}\left(M ; \mathbb{Z}_{8}\right.$ ), respectively (we could also write $\omega=\rho_{8} \widetilde{\omega}$, by the identity $i_{2} \circ \rho_{4}=$ $\rho_{8} \circ i_{2}$ ). By (5), we have

$$
\Gamma(\omega)=\rho_{4} \Gamma(\widetilde{\omega})=\overline{2} \in \mathbb{Z}_{4} .
$$

Note that our manifold $M$ has only one more nonzero invariant, independent of $H_{i}(M)$ and $\Gamma(M)$, namely, $\left\langle\alpha^{3},[M]\right\rangle=\overline{2}$.

Now we are going to describe a similar construction of another manifold $M_{1}$. This time we begin, instead of the knotted 3-sphere and the trivial normal framing, with the standard (unknotted) $S^{3} \subset S^{6}$ and the "index 2" framing (which corresponds to twice the generator of $\pi_{3} S O(3)$ ). Surgery along $S^{3}$ provides a spin manifold $\widetilde{M}_{1}$ with the same homology as $\widetilde{M}$ and with $\left\langle\widetilde{\alpha}^{3},\left[\widetilde{M}_{1}\right]\right\rangle=2$ and $\left\langle p_{1}\left(\widetilde{M}_{1}\right) \widetilde{\alpha},\left[\widetilde{M}_{1}\right]\right\rangle=8$ (again, see [10, Theorem 4]); in fact, our $\widetilde{M}_{1}$ is a "double $\mathbb{C} P(3)$ " - a connected sum of 2 copies of $\mathbb{C} P(3)$ along $\mathbb{C} P(1))$. Calculation by formula (2) gives $\Gamma\left(\widetilde{M}_{1}, \widetilde{\omega}\right)=0$. Then we proceed in quite the same way as above: killing 4 times the generator of $H_{2}\left(\widetilde{M}_{1}\right)$ yields a manifold $M_{1}$ such that $H_{i}\left(M_{1}\right), w_{2}\left(M_{1}\right), p_{1}\left(M_{1}\right)$, and $\left\langle\alpha^{3},\left[M_{1}\right]\right\rangle$ - essentially all the "conventional" 
invariants in our case - are the same as those of $M$. On the other hand, we have $\Gamma\left(M_{1}, \omega\right)=\overline{0} \neq \Gamma(M, \omega)$; hence $M$ and $M_{1}$ are not homeomorphic.

On the proof of the existence of the invariant $\Gamma$ in 2 . We begin with introducing a somewhat different view of our invariant. Let $G$ be a finitely generated Abelian group and $w: G \rightarrow \mathbb{Z}_{2}$ a homomorphism; the latter can also be regarded as a cohomology class, $w \in H^{2}\left(K(G, 2) ; \mathbb{Z}_{2}\right)$. Denote by $\Omega_{6}^{\text {spin }}(G, 2 ; w)$ the bordism group of maps $f$ : $M \rightarrow K(G, 2)$ satisfying the "spin condition" $f^{*}(w)=w_{2}(M)$, the group operation being the usual connected sum. (As above, here 6-manifolds $M$ are assumed to be simply connected: without this condition, one would have to fix some sort of a "spin structure" on the maps $M \rightarrow K(G, 2)$; see [2] for the details.) In a standard way, any class $\omega \in H_{\text {spin }}^{2}\left(M ; \mathbb{Z}_{2 a}\right)$ determines a homotopy class of maps $M \rightarrow K\left(\mathbb{Z}_{2 a}, 2\right)$, thereby a bordism class $[M, \omega] \in \Omega_{6}^{\mathrm{spin}}\left(\mathbb{Z}_{2 a}, 2 ; \rho_{2}\right)$. Since axiom $\Gamma 2$ implies that the value $\Gamma(M, \omega)$ depends only on the bordism class $[M, \omega]$, we obtain a map

$$
\Gamma_{a}: \Omega_{6}^{\mathrm{spin}}\left(\mathbb{Z}_{2 a}, 2 ; \rho_{2}\right) \rightarrow \mathbb{Z}_{a} .
$$

Now we observe that axiom $\Gamma 4$ determines the map $\Gamma_{0}: \Omega_{6}^{\mathrm{spin}}\left(\mathbb{Z}, 2 ; \rho_{2}\right) \rightarrow \mathbb{Z}$, while axiom $\Gamma 3$ requires that the diagram

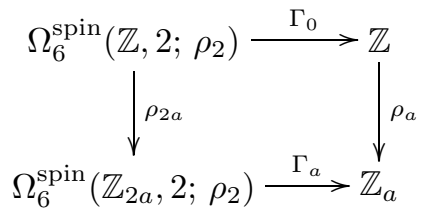

should be commutative. Note that the left vertical arrow in this diagram is an epimorphism (the proof is contained in the argument preceding formula (50), so that these two axioms $-\Gamma 3$ and $\Gamma 4-$ determine the family of homomorphisms (6), alias invariant $\Gamma$, provided the inclusions

$$
\operatorname{Ker}\left(\rho_{2 a}\right) \subset \operatorname{Ker}\left(\rho_{a} \circ \Gamma_{0}\right)
$$

are true. The groups $\Omega_{6}^{\mathrm{spin}}\left(\mathbb{Z}_{2 a}, 2 ; \rho_{2}\right)$, as well as more general groups $\Omega_{6}^{\mathrm{spin}}(G, 2 ; w)$, were calculated in [2, 11]. In particular, this calculation justifies the inclusions (8), and therefore the existence of $\Gamma$ (in fact, this is how $\Gamma$ was defined in [2, §5.17], i. e., by formula (2) and diagram (7)).

\section{§2. Direct COnstruction}

The construction of the "resolution manifold" $\widetilde{M}$ appearing in our fundamental formula (5) contained much uncertainty that is impossible to "track down" (otherwise we could simply use this formula as a definition); in a sense, what was said at the end of $\S 1$ may be regarded as putting this uncertainty in a more "manageable" form. Now we are going to give a version of this construction with "least possible" uncertainty, which will allow us to check directly, without referring to the bordism groups $\Omega_{6}^{\text {spin }}\left(\mathbb{Z}_{2 a}, 2 ; \rho_{2}\right)$, etc., that it defines an invariant consistently. For this, we introduce an auxiliary means: a class of 4-dimensional singular subsets in 6-manifolds representing nonintegral homology classes.

$\boldsymbol{\Sigma}_{n}$-submanifolds. For any $n \geq 2$, let $C_{n}$ denote a cone in the 3 -ball $D^{3}$ with vertex 0 (the center of the ball) over an $n$-point set $\left\{q_{1}, q_{2}, \ldots, q_{n}\right\}$ on the equator of the sphere $S^{2}$ (a particular arrangement of the points $q_{i}$ does not matter). Denote by $\Sigma_{n}$ the subset $S^{3} \times C_{n}$ of $S^{3} \times D^{3}$; this is a stratified set having one 4-dimensional stratum (the union 
of $n$ cylinders $\left.S^{3} \times(0,1)\right)$ and one 3 -dimensional stratum (the 3 -sphere). We give all strata the "product orientation", assuming all radii to be oriented "towards the center".

Now suppose we are given a compact stratified set $A$ in a 6 -manifold $M$, consisting of 2 oriented strata, the 4-dimensional stratum int $A$ and the 3 -dimensional stratum $\partial A$; suppose furthermore that near $\partial A$ the set $A$ coincides (orientations included) with $f\left(\Sigma_{n}\right)$ for some embedding $f: S^{3} \times D^{3} \rightarrow M$. We call such an $A$ a $\Sigma_{n}$-submanifold of $M$. We say also that $A$ is subordinate to any embedding $f$ as above. Note that, for a given $A$, an embedding $f$ can be chosen both to preserve and to reverse orientations (assuming $M$ oriented), which is evident from the existence of an orientation-reversing symmetry $D^{3} \rightarrow D^{3}$ that preserves also the cone $C_{n}$ (this remark will be used later).

Let $[A] \in H_{4}(A, \partial A)$ be the relative fundamental class; obviously, its homological boundary is $n[\partial A]$, so that after reducing modulo $n$ this becomes an absolute class $\rho_{n}[A] \in H_{4}\left(A ; \mathbb{Z}_{n}\right)$. We denote this reduced fundamental class, as well as its image in $H_{4}\left(M ; \mathbb{Z}_{n}\right)$, by $[A]_{n}$.

Theorem 3. If $M$ is simply connected, then for any class $x \in H_{4}\left(M ; \mathbb{Z}_{n}\right)$ there exists a $\Sigma_{n}$-submanifold $A \subset M$ with $[A]_{n}=x$.

Proof. Let $\beta: H_{4}\left(M ; \mathbb{Z}_{n}\right) \rightarrow H_{3}(M)$ be the homological Bokstein homomorphism. We take any embedding $f: S^{3} \times D^{3} \rightarrow M$ with $f_{*}\left[S^{3}\right]=\beta(x)$ (here we need the fact that $M$ is simply connected). Denote $f\left(S^{3} \times D^{3}\right)$ by $U$, and consider the homomorphisms $j_{*}$, $\rho_{n}$, and $\partial$ in the diagram

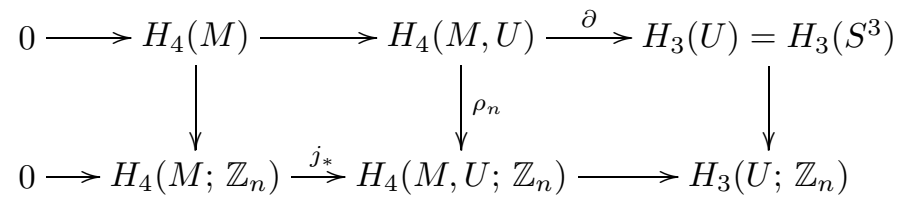

Since, clearly, $\beta\left(j_{*} x\right)=j_{*} \beta(x)=0$, the class $j_{*} x$ is integral: there exists $x_{0} \in H_{4}(M, U)$ with

$$
\rho_{n}\left(x_{0}\right)=x .
$$

Moreover, we can choose $x_{0}$ so as to satisfy the extra relation

$$
\partial\left(x_{0}\right)=n\left[f\left(S^{3}\right)\right] .
$$

Indeed, let $\xi$ be an integral chain representing the class $x$, and, in the same way, let a chain $s$ represent the class $\left[f\left(S^{3}\right)\right]$. The relation $\left[f\left(S^{3}\right)\right]=\beta(x)$ can be written on the chain level as $s=\frac{1}{n} \partial \xi+\partial \eta$, or alternatively as $\partial(\xi+n \eta)=n s$. Thus, $\xi+n \eta$ is a relative cycle giving the desired class $x_{0}$.

Now we apply excision: we put $M_{0}=M \backslash \operatorname{int} U$ and $S_{i}^{3}=f\left(S^{3} \times q_{i}\right) \subset \partial M_{0}$ (the points $q_{i}$ from the definition of $C_{n}$ above). Next, we rewrite (11) as

$$
\partial\left(x_{0}\right)=\left[\bigcup_{i=1, \ldots, n} S_{i}^{3}\right] .
$$

By Theorem 1 there exists a submanifold $A_{0} \subset M_{0}$ with $\partial A_{0}=\bigcup_{i} S_{i}^{3}$ and $\left[A_{0}\right]=x_{0}$. Now put $A=A_{0} \cup f\left(S^{3} \times C_{n}\right)$ (and perform angle-smoothing in the DifF case).

Signatures. With each oriented $\Sigma_{n}$-submanifold $A$ we can canonically associate its "unfolding" $A^{u}$ — an oriented 4-manifold whose boundary is $n$ disjoint copies of $S^{3}$ (in essence, this is $A_{0}$ of the above proof). Obviously, the intersection form of $A^{u}$ is nonsingular; its signature will also be referred to as the signature of $A$, and denoted accordingly by $\sigma(A)$. If $k=2 a$ and $[A]_{2 a} \in H_{4}^{\text {spin }}\left(M ; \mathbb{Z}_{2 a}\right)$, then $A^{u}$ is a spin manifold, so that $\sigma(A)$ is divisible by 8 . The following theorem may be regarded as an extension of our normalization axiom $\Gamma 4$ to nonintegral classes $\omega$. 
Theorem 4. Let a $\Sigma_{2 a}$-submanifold $A \subset M$ represent the homology class D $\omega$ with $\omega \in H_{\mathrm{spin}}^{2}\left(M ; \mathbb{Z}_{2 a}\right)$. Then

$$
\Gamma(\omega)=\rho_{a}\left(\frac{3}{8} \sigma(A)\right) .
$$

Proof. The idea is to eliminate the singular stratum $\partial A$ by surgery along $f$, and to apply axiom $\Gamma 2$. Let $\widetilde{M}=M_{0} \cup_{f}\left(D^{4} \times S^{2}\right)$ be the result of the surgery (we use the notation as in the proof of Theorem 3); denote by $\widetilde{A}$ the closed submanifold $A_{0} \cup_{f}\left(D^{4} \times\left\{q_{1}, \ldots, q_{2 a}\right\}\right)$ of $\widetilde{M}$ obtained by "filling up" the boundary spheres $S_{i}^{3}$. The fundamental class $[\widetilde{A}] \in H_{4}(\widetilde{M})$ will be denoted by $\widetilde{x}$ and its Poincaré dual by $\widetilde{\omega}$.

Let $W=(M \times[0,1]) \cup_{(f, 1)}\left(D^{4} \times D^{3}\right)$ be the standard cobordism between $M$ and $\widetilde{M}$. It is easily seen that there exists a class $\zeta \in H^{2}\left(W ; \mathbb{Z}_{2 a}\right)$ whose restrictions to $M$ and $\widetilde{M}$ are $\omega$ and $\rho_{2 a} \widetilde{\omega}$, respectively. Indeed, such a $\zeta$ can be described as the Poincaré dual $D^{-1} z$ of the relative homology class $z \in H_{5}\left(W, M \cup \widetilde{M} ; \mathbb{Z}_{2 a}\right)$ represented by the stratified set $(A \times[0,1]) \cup_{(f, 1)}\left(D^{4} \times C_{2 a}\right)$. By axioms $\Gamma 2$ and $\Gamma 3$, we now have $\Gamma(M, \omega)=\Gamma\left(\widetilde{M}, \rho_{2 a} \widetilde{\omega}\right)=\rho_{a} \Gamma(\widetilde{M}, \widetilde{\omega})$; together with the normalization axiom, this gives the required result.

Note that the above argument can be viewed as a certain "concretization" of that employed to prove formula (5): instead of killing the entire Tors $H_{3}(M)$, we now kill only one torsion element $\beta(D \omega)$, and instead of taking any integral class $\widetilde{\omega}$ "covering" a given $\omega$, we produce some specific $\widetilde{\omega}$ (actually, a cocycle).

The main theorem. From Theorem 4 along with the results of [2, it follows that the expression $\rho_{a}\left(\frac{3}{8} \sigma(A)\right)$ depends only on the homology class $[A]_{2 a}$. Now we are going to "invert" this: the statement below does not depend on [2], and allows us to define $\Gamma(\omega)$ by formula (12).

Theorem 5. For any simply connected closed 6-manifold $M$ and any $\Sigma_{2 a}$-submanifolds $A, A^{\prime} \subset M$ with $[A]_{2 a}=\left[A^{\prime}\right]_{2 a} \in H_{4}^{\text {spin }}\left(M ; \mathbb{Z}_{2 a}\right)$, we have the congruence $3 \sigma(A) \equiv 3 \sigma\left(A^{\prime}\right)$ $\bmod 8 a$.

The proof of this theorem will take several subsections. We need to "trace" the possible changes of a $\Sigma_{2 a}$-submanifold $A$ within a fixed class $x=[A]_{2 a}$. It will be useful to return again to our notation in the proof of Theorem 3 and consider 3 levels of changes, in accordance with what stays fixed.

1) Both the embedding $f: S^{3} \times D^{3} \rightarrow M$ and the relative homology class $x_{0} \in$ $H_{4}(M, U)=H_{4}\left(M_{0}, \partial M_{0}\right)$ are fixed; only the submanifold $A_{0} \subset M_{0}$ may vary, with preservation of the boundary $\partial A_{0}=\bigcup_{i} S_{i}^{3}$ and the homology class $\left[A_{0}\right]=x_{0}$.

2) The embedding $f$ is fixed; the class $x_{0}$ may vary, with preservation of relations (10) and (11).

3) Nothing is fixed except the given class $x$; the embedding $f$ may vary within the homology class $\beta(x)$.

Level 1 is straightforward: if $A_{0}, A_{0}^{\prime} \subset M_{0}$ are two submanifolds with the same boundary $\partial A_{0}=\partial A_{0}^{\prime}=\bigcup_{i} S_{i}^{3}$ and homology class $\left[A_{0}\right]=\left[A_{0}^{\prime}\right]=x_{0}$, then $A_{0}$ and $A_{0}^{\prime}$ are cobordant (with fixed boundary), so that $\sigma\left(A_{0}\right)=\sigma\left(A_{0}^{\prime}\right)$. This simple argument shows that a priori $3 \sigma(A)$ is a function of 3 pieces of data: the $\mathbb{Z}_{2 a}$-homology class $x$, the embedding $f$, and the relative $\mathbb{Z}$-homology class $x_{0}$; put, for example, $3 \sigma(A)=F\left(x, f, x_{0}\right)$. Both $f$ and $x_{0}$ depend on $x$, but also contain some extra information, and our further objective is to show that $F\left(x, f, x_{0}\right)$ does not actually depend (modulo $8 a$ at least) on this extra information. 
Of the two levels remaining, number 3 looks most troublesome at first sight. Of course, the trivial case occurs here, represented by two isotopic embeddings $f, f^{\prime}: S^{3} \times D^{3} \rightarrow$ $M$. However, in general, the two embeddings in question may happen to be not only nonisotopic, but even nonhomotopic, and it seems absolutely nonevident how one should compare them. As we shall see below, one can circumvent this by a simple (and rather curious) argument involving non-simply-connected 6 -manifolds. Now we turn to level 2 , which needs some calculations.

Wu formulas. There are well-known Wu formulas "modulo 2" for Stiefel-Whitney classes; also, there are Wu formulas "modulo 4" [12] involving Pontryagin classes. We shall need the following two relations derived from some of these formulas.

Lemma 6. Given a closed orientable 6-manifold $M$ and integral cohomology classes $z \in H^{2}(M)$ and $\omega \in H_{\text {spin }}^{2}(M)$, we have the following congruences:

(a) $\left\langle\omega z^{2},[N]\right\rangle \equiv 0 \bmod 2$,

(b) $\left\langle\left(p_{1}+\omega^{2}\right) z,[N]\right\rangle \equiv 0 \bmod 4$.

Proof. The first congruence follows immediately from the relation

$$
\left\langle v_{2} z^{2},[N]\right\rangle=\left\langle\mathrm{Sq}^{2}\left(z^{2}\right),[N]\right\rangle=\left\langle\left(\mathrm{Sq}^{1} z\right)^{2},[N]\right\rangle=0,
$$

where $v_{2}=w_{2}=\rho_{2}(\omega)$ is the 2-dimensional $\mathrm{Wu}$ class.

For the second congruence we use one of the "modulo 4" Wu formulas [12]:

$$
\rho_{4}\left(p_{1}\right)=P\left(w_{2}\right)+i_{2}\left(w_{4}\right)
$$

(here $P: H^{2}\left(B S O ; \mathbb{Z}_{2}\right) \rightarrow H^{4}\left(B S O ; \mathbb{Z}_{4}\right)$ is the "Pontryagin square" nonstable cohomology operation [13]), plus yet another modulo 2 relation

$$
w_{4}+w_{2}^{2}=v_{4}=0,
$$

where $v_{4}$ is the 2-dimensional Wu class (identity (13) is universal, while (14) is valid for any orientable closed manifold of dimension less than 8). Replacing $w_{4}$ in (13) with $w_{2}^{2}$, we obtain

$$
\rho_{4}\left(p_{1}\right)=P\left(w_{2}\right)+i_{2}\left(w_{2}^{2}\right)=P\left(w_{2}\right)+2 P\left(w_{2}\right)=-P\left(w_{2}\right)=-\rho_{4}\left(\omega^{2}\right) .
$$

(Relation (b) can also be deduced from the following consequence of Rokhlin's "divisibility by 16 " theorem:

$$
\left\langle p_{1}(\omega+2 z)-(\omega+2 z)^{3},[M]\right\rangle \equiv 0 \bmod 16 ;
$$

see also [5]. We do not consider this in detail.)

Remark 4. Needless to say, "modulo 2" Wu formulas, hence the above proof for (a), work in the Top category. To extend (b), we need relation (13). Since the kernel of the natural homomorphism $H^{4}\left(B S\right.$ Top $\left.; \mathbb{Z}_{4}\right) \rightarrow H^{4}\left(B S O ; \mathbb{Z}_{4}\right)$ is generated by the class $i_{2} \Delta$, in any case we can write

$$
\rho_{4}\left(p_{1}\right)=P\left(w_{2}\right)+i_{2}\left(w_{4}\right)+t \cdot i_{2} \Delta
$$

with $t=0$ or $t=1$. Now, let $A$ be a closed simply connected topological 4-manifold with $w_{2}(A)=0$ and $\sigma(A)=8$; "evaluating" the left-hand side of (15) at $A$, we get 0 , and the right-hand side yields $t \cdot i_{2} \Delta(A)$, with $\Delta(A) \neq 0$. Hence $t=0$, which proves (b).

Proof of Theorem 5 (level 2). Let $x_{0}, x_{0}^{\prime} \in H_{4}(M, U)$ be relative classes occurring in the proof of Theorem [3, i. e., satisfying (10) and (11) (with $k=2 a$ ). Then the difference $u=x_{0}^{\prime}-x_{0}$ satisfies $\rho_{2 a}(u)=0$; therefore, it is divisible by $2 a$. Thus, we can write 
$x_{0}^{\prime}=x_{0}+2 a y$ for some $y \in H_{4}(M, U)$. Now, let $\widetilde{M}$ be the result of surgery, as in the proof of Theorem 4 , Note that the group $H_{4}(\widetilde{M})$ naturally identifies with $H_{4}(M, U)$ by

$$
H_{4}(M, U)=H_{4}\left(M \cup e^{4}\right)=H_{4}(W)=H_{4}\left(\widetilde{M} \cup e^{3}\right)=H_{4}(\widetilde{M})
$$

(this identification occurred in the proof of Theorem 4 under the title "filling up boundary spheres"). Choosing some orientation of $\widetilde{M}$ and denoting the Poincaré duals of $x_{0}, x_{0}^{\prime}, y$ by $\omega, \omega^{\prime}, z \in H^{2}(\widetilde{M})$, we have $\omega^{\prime}=\omega+2 a z$, so that the congruence to be proved takes the form

$$
p(\omega+2 a z)-p(\omega) \equiv 0 \quad \bmod 8 a
$$

(for any $z \in H^{2}(\widetilde{M}), \omega \in H_{\text {spin }}^{2}(\widetilde{M})$ ). In turn, this is established by an elementary check on the basis of formula (11) and Lemma 6 .

It is easily seen that, in fact, we have proved the following somewhat more general statement.

Theorem 7. Let $N$ be a closed orientable 6-manifold and $f: S^{3} \times D^{3} \rightarrow N$ an embedding. Let $A, A^{\prime} \subset N$ be $\Sigma_{2 a}$-submanifolds, both subordinate to $f$ and representing one and the same class $x \in H_{4}^{\text {spin }}\left(N ; \mathbb{Z}_{2 a}\right)$. Then $3 \sigma(A) \equiv 3 \sigma\left(A^{\prime}\right) \bmod 8 a$.

Note that the above conditions imply $[\partial A]=\left[\partial A^{\prime}\right]=\beta(x)$, so that our $\Sigma_{2 a}$-submanifolds can both be obtained by precisely the same process as in the proof of Theorem 3 . We emphasize that $N$ here may fail to be simply connected, which will be used immediately.

Proof of Theorem 5 (level 3). Again, let $M$ be simply connected, and let $\Sigma_{2 a}$-submanifolds $A, A^{\prime} \subset M$ satisfy the condition

$$
[A]_{2 a}=\left[A^{\prime}\right]_{2 a}=x \in H_{4}^{\mathrm{spin}}\left(M ; \mathbb{Z}_{2 a}\right) .
$$

Let $f, f^{\prime}: S^{3} \times D^{3} \rightarrow M$ be embeddings, and suppose that $A, A^{\prime}$ are subordinate to $f, f^{\prime}$, respectively. Now we are going to modify the embeddings $f, f^{\prime}$ and the ambient manifold $M$, at the same time preserving the topology of $A, A^{\prime}$ unchanged. Recall (see the remark after the definition of a $\Sigma_{n}$-submanifold) that we may assume $f, f^{\prime}$ to behave oppositely with respect to orientations.

Since the spheres $f\left(S^{3} \times\{0\}\right)$ and $f^{\prime}\left(S^{3} \times\{0\}\right)$ (both representing the same class $\beta(x)$ ) have zero intersection number, we can use the Whitney trick to make the sets $U=$ $f\left(S^{3} \times D^{3}\right)$ and $U^{\prime}=f^{\prime}\left(S^{3} \times D^{3}\right)$ disjoint.

Now we "squeeze out" neighborhoods of the singular strata, i. e., intersections $A \cap \operatorname{int} U$ and $A^{\prime} \cap \operatorname{int} U^{\prime}$, to the boundaries of $U$ and $U^{\prime}$. Clearly, it suffices to perform a similar procedure with the set $C_{2 a} \subset D^{3}$; for example, one can choose a point $c \in D^{3}$ outside the equatorial plane and apply the radial projection $D^{3} \backslash\{c\} \rightarrow S^{2}$ (we leave out the trivial problem of smoothing angles for the DIFF category).

Finally, we do surgery on $M$, namely, cut out the interiors of $U, U^{\prime}$ and identify the boundaries:

$$
M_{0}=M \backslash\left(\operatorname{int} U \cup \operatorname{int} U^{\prime}\right), \quad N=M_{0} / f(x) \in \partial U \sim f^{\prime}(x) \in \partial U^{\prime} .
$$

After this surgery, the sets $A, A^{\prime}$ become identical near the singular stratum. Moreover, it is easily seen that the embedding $M_{0} \rightarrow M$ induces an isomorphism $H_{4}\left(M_{0} ; \mathbb{Z}_{2 a}\right) \rightarrow$ $H_{4}\left(M ; \mathbb{Z}_{2 a}\right)$, so that (16) stays true after the surgery. Hence, the assumptions of Theorem 7 are satisfied (note that $\pi_{1}(N) \approx \mathbb{Z}$ ), and the proof of Theorem 5 is complete.

Now, finally, we can give our direct definition (independent of [2]) of the invariant $\Gamma$. 
Definition 8. For any simply connected closed oriented 6-manifold $M$, we define $\Gamma(M)$ to be the family of functions

$$
\Gamma_{a}(M): H_{\mathrm{spin}}^{2}\left(M ; \mathbb{Z}_{2 a}\right) \rightarrow \mathbb{Z}_{a}, a \in \widehat{\mathbb{N}},
$$

given for $a=0$ by formula (21), and for $a>0$ by formula (12).

\section{§3. Direct Definition and the Properties of $\Gamma$}

Definition 8 could be considered as logically perfect if we could prove, within its context, all the basic properties of $\Gamma$ (i.e., axioms $\Gamma 1-\Gamma 4$ ). Of course, now $\Gamma 1$ and $\Gamma 4$ follow "by definition"; also, axiom $\Gamma 3$ can easily be checked directly. Regretfully, the most important axiom $\Gamma 2$ seems inaccessible within our elementary approach. It turns out, however, that , starting with Definition 8, we can prove our "fundamental formula" (5), which, in its turn, implies all other properties of $\Gamma$ listed in $\$ 1$, except axiom $\Gamma 2$, and which suffices for explaining the examples given there. We begin with introducing, for a certain technical convenience, a slightly more general type of singular submanifolds than was done in $\S 2$.

Multi- $\Sigma_{n}$-submanifolds. By a multi- $\Sigma_{n}$-submanifold we understand a stratified set $A \subset M$ that differs from $\Sigma_{n}$-submanifolds in that its singular stratum $\partial A$ may consist of several $\Sigma_{k}$-components, where $k$ should be a multiple of $n$ (generally, different multiples for different components). If the number of components of the singular stratum (counting multiplicities) equals $m$, then we say that $A$ is an $m \Sigma_{n}$-submanifold. Like $\Sigma_{n}$-submanifolds, each $m \Sigma_{n}$-submanifold has its reduced fundamental class $[A]_{n} \in$ $H_{4}\left(M ; \mathbb{Z}_{n}\right)$, the unfolding $A^{u}$ (a compact oriented manifold with boundary consisting of $m n$ spheres) and the signature $\sigma(A)$. Along with the unfolding $A^{u}$, we shall use the notation $\widetilde{A}$ (already used above in the proof of Theorem 4) for a manifold obtained from $A^{u}$ by "filling up" the boundary spheres (note that $\widetilde{A}$ is uniquely determined in the DIFF category; see [14]).

The following lemma says that our direct definition of the invariant $\Gamma$ is "insensitive" to this generalization.

Lemma 9. For any $m \Sigma_{n}$-submanifold $A$ in a connected 6 -manifold $M$, there exists a $\Sigma_{n}$-submanifold $A^{\prime}$ with $\left[A^{\prime}\right]_{n}=[A]_{n}$ and $\sigma\left(A^{\prime}\right)=\sigma(A)$.

Proof. We begin with splitting each of the "multicomponents" of the boundary (i.e., those having multiplicity $k n$ with $k>1$ ), should these exist, into $k$ usual $\Sigma_{n}$-components (Figure 1 shows clearly how this works). Obviously, this procedure does not change the unfolding $A^{u}$. Now we are going to modify the submanifold $A$ and reduce the number of

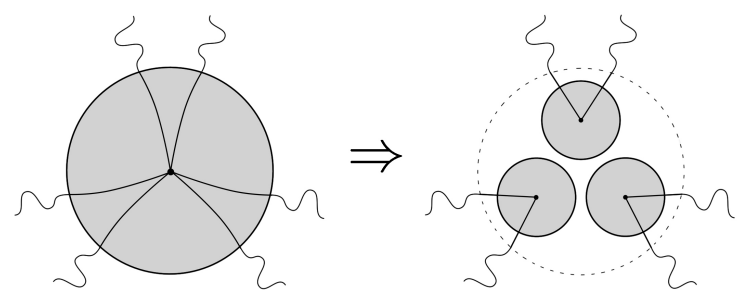

FiguRE 1

boundary components. We choose any two components $S_{0}, S_{1} \subset \partial A$ and join them with a "ribbon" $B \subset M$, which is the image of an embedding $f: D^{3} \times[0,1] \rightarrow M$ whose only intersections with $A$ are $B_{0}=B \cap S_{0}=f\left(D^{3} \times 0\right)$ and $B_{1}=B \cap S_{1}=f\left(D^{3} \times 1\right)$ 


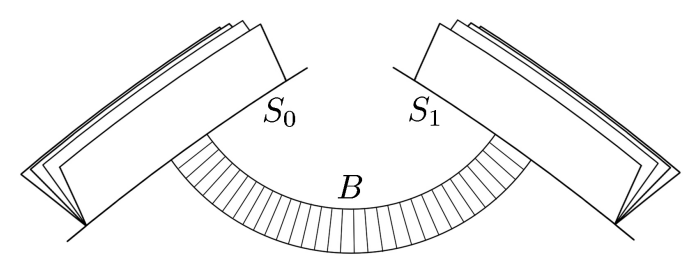

FiguRe 2

(see Figure 2). We can assume that the orientations of the components $S_{0}$ and $S_{1}$ match those of $B_{0}$ and $B_{1}$ (as induced from the standard orientation of $D^{3} \times[0,1]$ ). What we are going to do next could be called "ribbon cloning": choosing a normal field $v$ along $B$ and a set of sufficiently small numbers $\varepsilon_{1}<\varepsilon_{2}<\cdots<\varepsilon_{n}$, we replace the original embedding $f$ with $n$ embeddings $f_{1}, f_{2}, \ldots, f_{n}$ given by the formula

$$
f_{i}(x, t)=f(x, t)+\varepsilon_{i} \cdot\left(1-|x|^{2}\right) \cdot v
$$

with $x \in D^{3}$ and $t \in[0,1]$. Instead of the original ribbon, we obtain a "bundle of ribbons" with the same "side surface" $f: S^{2} \times[0,1]$ (see Figure 3, where $k=4$ ).

Now we "cut out" from $A$ some neighborhoods of $B_{0}$ and $B_{1}$, so that instead of each of these balls we get a "bundle of balls" with common boundary, and we paste the ends of our "ribbon bundle" to these bundles (see the right-hand side of Figure 3). We leave

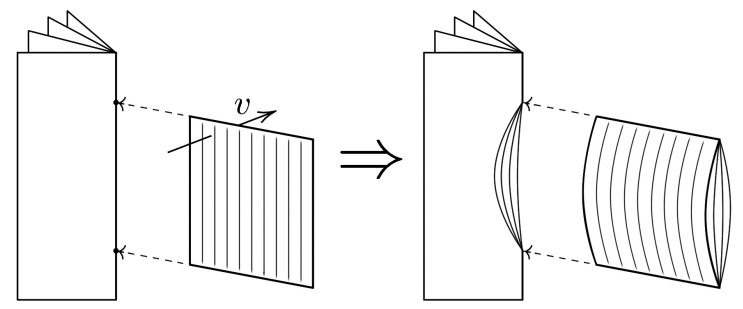

FiguRE 3

out a tiresome, however quite trivial, angle-smoothing procedure for DifF. The result of this process is a new multi- $\Sigma_{n}$-submanifold, say $A_{1}$, having one component less than the original $A$. Obviously, $\left[A_{1}\right]_{n}=[A]_{n}$. Also, it is easily seen that $\sigma\left(A_{1}\right)=\sigma(A)$; indeed, the unfolding $A_{1}^{u}$ is obtained from $A^{u}$ by attaching several 1-handles to $\partial A^{u}$, which does not affect the 2-homology.

To complete the proof, we simply repeat this process until there is only one boundary 3 -sphere left.

Remark 5. To adapt the above argument for ToP, one can use the "local product structure theorem" [7, Essay I, Theorem 5.2] to extend the smooth structures on the neighborhoods of $S_{0}$ and $S_{1}$ to a sequence of coordinate charts connecting these neighborhoods, thus finding oneself back in the DIFF category.

Let $\widetilde{M}$ be a "resolution manifold" for $M$. Recall that $\widetilde{M}$ is obtained from $M$ by a series of surgeries along some embeddings $f_{i}: S^{3} \times D^{3} \rightarrow M$ that realize a basis for the group Tors $H_{3}(M)$. Also, we have natural inclusions $H^{2}(M ; G) \subset H^{2}(\widetilde{M} ; G)$ (for any coefficients $G)$, which we put here in dual form as $H_{4}(M ; G) \subset H_{4}(\widetilde{M} ; G)$. The 
following theorem is, so to speak, a "universalization" of the argument used above to derive formula (12).

Theorem 10. Each class $x \in H_{4}\left(M ; \mathbb{Z}_{n}\right)$ may be represented by a multi- $\Sigma_{n}$-submanifold $A \subset M$ such that the corresponding $\widetilde{A}$ is canonically embedded in $\widetilde{M}$ and represents there an integral homology class reducing modulo $n$ to the class $x$.

Proof. We need a minor complication of a construction used in the proof of Theorem 3 , Denoting the set $\bigcup_{i} f_{i}\left(S^{3} \times D^{3}\right) \subset M$ by $U$, we get the same diagram (9). Reproducing verbatim the argument that follows, we obtain a class $x_{0} \in H_{4}(M, U)$ with $\rho_{n}\left(x_{0}\right)=x$ and $\partial\left(x_{0}\right)=\sum_{i} n a_{i} \cdot\left[f_{i}\left(S^{3}\right)\right]$, where the $a_{i}$ are coefficients for the expansion of $\beta(x)$ in the basis $\left\{\left[f_{i}\left(S^{3}\right)\right]\right\}$ (clearly we may assume that $a_{i} \geq 0$ ). Applying excision and Theorem 1 precisely as in the proof of Theorem 3, we get the desired $m \Sigma_{n}$-submanifold (with $m=\sum_{i} a_{i}$ ); the construction of an embedding $\widetilde{A} \subset \widetilde{M}$ does not differ from that in the proof of Theorem 4 .

Proof of formula (5). We have the following "input data": a simply connected closed oriented manifold $M$, a "resolution manifold" $\widetilde{M}$, and cohomology classes $\omega \in H_{\text {spin }}^{2}\left(M ; \mathbb{Z}_{2 a}\right)$ and $\widetilde{\omega} \in H_{\text {spin }}^{2}(\widetilde{M})$ with $\rho_{2 a}(\widetilde{\omega})=\omega$ (recall again that we view the groups $H^{2}(M ; G)$ as canonically embedded in $\left.H^{2}(\widetilde{M} ; G)\right)$.

Let $A \subset M$ be a multi- $\Sigma_{n}$-submanifold realizing the class $D \omega$, whose existence is stated in Theorem 10, Denote by $\widetilde{\omega}_{0} \in H_{\text {spin }}^{2}(\widetilde{M})$ the class dual to $[\widetilde{A}] \in H_{4}(\widetilde{M})$. By Definition 8 and Lemma 9 (and also by $\sigma(A)=\sigma(\widetilde{A})$ ), we have the chain of identities

$$
\Gamma(M, \omega)=\rho_{a}\left(\frac{3}{8} \sigma(A)\right)=\rho_{a}\left(\frac{3}{8} \sigma(\widetilde{A})\right)=\rho_{a} \Gamma\left(\widetilde{M}, \widetilde{\omega}_{0}\right) .
$$

On the other hand, $\rho_{2 a}\left(\widetilde{\omega}_{0}\right)=\rho_{2 a}(\widetilde{\omega})$, whence

$$
\Gamma\left(\widetilde{M}, \widetilde{\omega}_{0}\right) \equiv \Gamma(\widetilde{M}, \widetilde{\omega}) \bmod a
$$

(see the proof of level 2 in Theorem [5). Combining (17) and (18) completes the proof.

\section{REFERENCES}

[1] A. V. Zhubr, Classification of simply-connected topological 6-manifolds, Topology and GeometryRohlin Seminar, Lecture Notes in Math., vol. 1346, Springer, Berlin, 1988, pp. 325-339. MR0970082 (89k:57065)

[2] Closed simply connected 6-manifolds: the proofs of classification theorems, Algebra i Analiz 12 (2000), no. 4, 126-230; English transl., St. Petersburg Math. J. 12 (2001), no. 4, 605-680. MR:1793619 (2001j:57041)

[3] F. Quinn, Ends of maps. III. Dimensions 4 and 5, J. Differential Geom. 17 (1982), 503-521. MR0679069 (84j:57012)

[4] F. Hirzebruch, Topological methods in algebraic geometry, Grundlehren Math. Wiss., Bd. 131, Springer-Verlag New York, Inc., New York, 1966. MR.0202713 (34:2573)

[5] P. E. Jupp, Classification of certain 6-manifolds, Proc. Cambridge Philos. Soc. 73 (1973), 293-300. MR0314074 (47:2626)

[6] L. C. Siebenmann, Topological manifolds, Actes du Congrès International des Mathématiciens (Nice, 1970), T. 2, Gauthier-Villars, Paris, 1971, pp. 133-163. MR0423356 (54:11335)

[7] R. C. Kirby and L. C. Siebenmann, Foundational essays on topological manifolds, smoothings, and triangulations, Ann. Math. Stud., No. 88, Princeton Univ. Press, Princeton, NJ; Univ. Tokyo Press, Tokyo, 1977. MR0645390 (58:31082)

[8] M. H. Freedman, The topology of four-dimensional manifolds, J. Differential Geom. 17 (1982), 357-453. MR0679066 (84b:57006)

[9] A. Haefliger, Knotted (4k-1)-spheres in 6k-space, Ann. of Math. (2) $\mathbf{7 5}$ (1962), 452-466. MR0145539(26:3070)

[10] C. T. C. Wall, Classification problems in differential topology. V. On certain 6-manifolds, Invent. Math. 1 (1966), 355-374. MR0215313 (35:6154) 
[11] A. V. Zhubr, Spin bordism of oriented manifolds and the Hauptvermutung for 6-manifolds, Topology, Ergodic Theory, Real Algebraic Geometry (V. Turaev, A. Vershik, eds.), Amer. Math. Soc. Transl. Ser. 2, vol. 202, Amer. Math. Soc., Providence, RI, 2001, pp. 263-286. MR1819194 (2002b:57034)

[12] Wen-tsün Wu, On Pontrjagin classes. III, Acta Math. Sinica 4 (1954), 323-346. MR0080300 $(18: 225 \mathrm{f})$

[13] R. Mosher and M. Tangora, Cohomology operations and applications in homotopy theory, Harper and Row, New York-London, 1968. MR0226634 (37:2223)

[14] J. Cerf, Sur les difféomorphismes de la sphère de dimension trois $\left(\Gamma_{4}=0\right)$, Lecture Notes in Math., vol. 53, Springer-Verlag, Berlin-New York, 1968. MR 0229250 (37:4824)

Mathematics Department, Komi Scientific Center, Urals Division, Russian Academy of Sciences, Chernova Street 3A, Syktyvkar 167998, Russia

E-mail address: a-v-zhubr@yandex.ru

Received 20/MAY/2008

Translated by THE AUTHOR 